\title{
Deficiency of knowledge of genetics and genetic tests among general practitioners, gynecologists, and pediatricians: A global problem
}

\author{
Marieke J.H. Baars, $M D^{1}$ Lidewij Henneman, $P h D^{1,2}$, and Leo P. ten Kate, MD, $P h D^{1}$
}

\begin{abstract}
Purpose: The objective of this study was to assess knowledge of genetics and awareness of genetic tests among Dutch general practitioners (GPs), gynecologists (GYNs), and pediatricians (PEDs), as well as factors influencing their knowledge and awareness. Methods: An anonymous questionnaire inquiry was used, validated with a sample of 52 clinical geneticists (CGs). The study was carried out in primary care (general practice) and secondary care (general and university hospitals) in The Netherlands. A random sample of 200 GPs, 300 GYNs, and 265 PEDs received a questionnaire. In addition, all registered CGs (58) received a questionnaire for validation. In total, 122 GPs, 187 GYNs, 164 PEDs, and 52 CGs returned a completed questionnaire. The main outcome measures were differences in knowledge scores between physicians working in different disciplines and factors influencing these scores. Results: Knowledge scores of GPs (mean 64\% correct answers, 61\%-66\% [95\% confidence interval]), GYNs (mean $75 \%$ correct answers, $73 \%-76 \%$ [95\% confidence interval]), and PEDs (mean $81 \%$ correct answers, $79 \%-82 \%$ [95\% confidence interval]) were lower than those in the CG validation group (mean 95\% correct answers, 94\%-96\% [95\% confidence interval]). The 5th percentile of GPs, GYNs, and PEDs was at approximately $40 \%, 52 \%$ and $62 \%$ correct answers, respectively. There was a specific lack of knowledge about DNA testing. In addition to specialty, important factors positively associated with the knowledge scores of nongeneticists are more recent graduation, having taken an elective course in genetics, and providing genetic counseling in their own practice. Conclusion: The overall knowledge levels of genetics in many nongeneticist health care providers show clear deficiencies. This is in line with reports from other countries, showing that these deficiencies are a global problem.
\end{abstract}

Genet Med 2005:7(9):605-610.

Key Words: genetics, knowledge, general practitioners, gynecologists, pediatricians

Recent mapping of the Human Genome and the discovery of an increasing number of genes involved in the development of diseases will result in the availability of an increasing number of genetic tests in daily clinical practice. ${ }^{1-3}$ As nongeneticists will play a major role in ordering these tests, interpreting their results, referring patients to genetic centers, and communicating with patients about genetic issues, their knowledge of genetics becomes an essential part of good clinical practice. This requires, at least, sufficient knowledge about genetics and genetic tests. In many European countries GPs are the primary care providers and act as gatekeepers in the referral of patients to specialist care. ${ }^{4}$

\footnotetext{
From the ${ }^{1}$ Department of Clinical Genetics and Human Genetics, and ${ }^{2}$ Department of Public and Occupational Health, Institute for Research in Extramural Medicine (EMGO Institute), VU University Medical Center, Amsterdam, The Netherlands.

Marieke J.H. Baars, MD, Department of Clinical Genetics and Human Genetics, VU University Medical Center, P.O. Box 7057, 1007 MB Amsterdam, The Netherlands.

Submitted for publication May 3, 2005.

Accepted for publication August 8, 2005.
}

DOI: 10.1097/01.gim.0000182895.28432.c7
Until now, only a limited number of studies have investigated physicians' knowledge about genetics and their awareness of genetic tests. ${ }^{5-9}$ Hofman et al. ${ }^{5}$ showed that deficiencies still remain, despite the increasing knowledge of genetics among recently physicians who recently graduated in the United States. Hunter et al. ${ }^{6}$ showed that although a majority of Canadian physicians (family physicians, obstetricians, pediatricians [PEDs], and internists) considered their knowledge of genetics to be adequate, only a minority of them were confident in their ability to provide genetic counseling for simple scenarios. British general practitioners (GPs) currently believe that they lack the relevant knowledge and skills to manage patients who are concerned about their family history, according to Watson et al. ${ }^{7}$ In a systematic review by Suther et al. ${ }^{10}$ it was found that only 3 of 18 studies incorporated in the study validated their survey instrument or assessed the scores' reliability. One of the conclusions of that study is that further studies are needed to validate the finding of earlier studies and to establish their generalizability to various populations of primary care providers, especially in nations other than the United Kingdom and United States. The objective of this study is to assess knowledge of genetics and awareness of genetic tests in ran- 
dom samples of GPs, gynecologists (GYNs), and PEDs in The Netherlands using a validated survey instrument.

\section{MATERIALS AND METHODS}

\section{Survey instrument}

To investigate physicians' knowledge of genetics and genetic tests, a validated questionnaire designed by Hofman et al. ${ }^{5}$ was used, with their permission. This questionnaire was translated into Dutch and adapted to the health care system in The Netherlands. The Dutch version of the questionnaire was validated by translation back into English, and the text was revised where necessary. After a pretest on five GPs, the final version was compiled. In general, a multiple choice, yes-no, or matching format was used, ${ }^{5}$ and it took approximately 30 minutes to complete.

The questionnaire contained, among others, 26 knowledge questions consisting of 9 fact questions and 17 concept questions. The 9 fact questions included 2 questions on the characteristics of genetic disorders and 7 questions on the availability of genetic tests for specific diseases; the 17 questions about genetic concepts consisted of 6 questions about pedigree, 5 questions on familiarity with the concept of probability, and 6 questions about understanding genetic terms.

In one section of the questionnaire, dealing with cystic fibrosis $(\mathrm{CF})$, respondents were asked to remove a sticker after answering certain questions and then read the additional information underneath. They needed this additional information to base their subsequent answers on accurate information. Results of the part of the questionnaire dealing with the opinions of physicians with regard to preconceptional CF carrier screening ${ }^{11}$ and genetic testing in general, and their self-reported skills, are described elsewhere (Baars et al., unpublished, 2005).

Several questions on variables potentially explaining differences in knowledge levels were also included. Potential variables in all three specialties (GPs, GYNs, and PEDs) were gender, age, years of experience, year of graduation, genetics in the medical curriculum, having attended these required lessons, having taken an elective course in genetics during medical school, faculty membership, involvement in fellow education, opinion on the importance of diagnosing hereditary disorders, discussion of hereditary background with patients, frequency of seeing patients with a "hereditary disease," provision of genetic counseling in own practice, personal or family experience with genetic counseling, source of information generally used to keep up to date, referral for genetic counseling, and innovativeness (willingness to offer predictive genetic tests when this is not common practice). ${ }^{12}$ Specific variables were practice characteristics and involvement in obstetrics for the GPs, working in a university or general hospital for the GYNs and PEDs, and membership in the Section on Congenital and Inherited Disorders (SCID) of the Pediatric Association of The Netherlands for the PEDs.

\section{Validation}

In 2000, all active members $(\mathrm{N}=58)$ of the Dutch Society of Clinical Genetics received a version of the questionnaire containing only the 26 knowledge questions.

\section{Sample}

A random sample of 200 of the 7106 GPs practicing in The Netherlands, selected by The Netherlands Institute of Health Services Research, received the questionnaire in 2000. A random sample of 300 of the 691 practicing members of the Dutch Society of Obstetrics and Gynecology received the questionnaire in 2001. Only GYNs who were involved in obstetrics were included because the others were not expected to be confronted with questions from patients about the topics on the questionnaire. The practicing members of the Pediatrics Association of The Netherlands were divided into two groups: members of the SCID $(\mathrm{N}=65)$ and other members $(\mathrm{N}=804)$. All 65 members of the SCID and a random sample of 200 from the remaining 804 members received the questionnaire in 2001.

\section{Procedures}

The questionnaires were sent by mail. The follow-up included a brief postal reminder 1 week later, a second postal reminder after 1 month, enclosing the questionnaire again, and a phone-call reminder from the investigator after 2 months (only for GPs because of their lower response rate). An incentive of 20 Euros was offered to all respondents except to clinical geneticists (CGs).

\section{Analysis}

By assigning 1 point for each correct answer, an overall knowledge score was created with a maximum total score of 26 points (total knowledge score). Two subscores were calculated, one for the fact questions ("fact score") and one for the concept questions ("concept score"). The maximum subscores were 9 and 17 points, respectively. All analyses were performed in SPSS 9.0 for Windows (SPSS Inc., Chicago, IL). Reliability of the test was estimated with Cronbach's alpha. Differences between the mean knowledge scores of the PEDs who were SCID members and the other members of the Pediatrics Association were tested with the $t$ test for continuous data. Because no statistically significant difference was found between the knowledge scores and potentially explanatory variables for the PEDs who were SCID members and the other members of the Pediatrics Association of The Netherlands, the two groups were combined in further analysis. Differences between the mean knowledge scores of the various groups of specialties (GPs, GYNs, and PEDs) and CGs, as well as between the various specialties, were tested using one-way analysis of variance with post hoc Tukey's honestly significant test correction for multiple comparisons. Correlations were analyzed by calculating Spearman's rho coefficient. Simple linear correlations were used to find variables influencing the knowledge scores within each specialty. Age, years of experience, and year of graduation 
were all highly correlated, and therefore considered as one variable (i.e., year of graduation as a medical doctor).

To adjust for covariance, variables with simple linear correlations with knowledge and $P$ values of less than .1 were included in a multivariate linear regression analysis, with knowledge score of each specialty as the dependent variable. Overall, a $P$ value less than .05 was considered to be statistically significant (two-sided).

\section{RESULTS}

\section{Response rate and demographic characteristics}

Of the 200 GPs, 300 GYNs, and 265 PEDs, 5, 5, and 18, respectively, were no longer working, and were therefore excluded, as were 10 GYNs who were no longer working in the field of obstetrics. The response rate was 64\% for GPs (124/ 195), 69\% for GYNs (198/285), and 72\% for PEDs (177/247). Some respondents did not answer all the questions. For that reason, only questionnaires from respondents who answered more than $75 \%$ of all questions and also answered all knowledge questions were included in the analysis. Further analyses were thus restricted to the answers from 122 GPs, 187 GYNs, and 164 PEDs. In the validation group, 90\% of CGs (52/58) returned the questionnaire. Cronbach's alpha for a random sample $(\mathrm{n}=100)$ of the responding GPs, GYNs, and PEDs ( $\mathrm{n}$ $=473$ ) was 0.62 .

Table 1 shows the sociodemographic characteristics of the nongeneticists who were included in further analysis and their genetic education. The mean age and years of experience were within the expected range, considering the time since graduation. Most GPs (86\%) were not involved in obstetrics, and a minority of the GYNs and PEDs were working in university hospitals.

Table 1

Sociodemographic characteristics and their genetic education of the nongeneticist responders

\begin{tabular}{lccc}
\hline & $\begin{array}{c}\text { GPs } \\
\mathrm{N}=122\end{array}$ & $\begin{array}{c}\text { GYNs } \\
\mathrm{N}=187\end{array}$ & $\begin{array}{c}\text { PEDs } \\
\mathrm{N}=164\end{array}$ \\
\hline Male (\%) & 81 & 75 & 59 \\
Age $<40$ y (\%) & 21 & 16 & 23 \\
Age $40-54$ y (\%) & 69 & 66 & 62 \\
Age $>54$ y (\%) & 10 & 18 & 15 \\
$\begin{array}{l}\text { Years of experience as a } \\
\text { specialist (mean, median, } \\
\text { range })^{a}\end{array}$ & $16,16,34$ & $13,14,29$ & $13,12,30$ \\
$\begin{array}{l}\text { Working in a university hospital } \\
(\%)\end{array}$ & - & & \\
$\begin{array}{l}\text { Genetics in the medical } \\
\text { curriculum (yes, \%) }\end{array}$ & & 16 & 38 \\
$\begin{array}{l}\text { Having taken an elective } \\
\text { course in genetics } \\
\quad(\text { yes, \%) }\end{array}$ & 84 & 71 & 76 \\
\hline
\end{tabular}

${ }^{a}$ Range (maximum minus minimum).

GP, general practitioner; GYN, gynecologist; PED, pediatrician.

\section{Genetic knowledge}

Table 2 shows mean, 95\% confidence intervals, standard deviation, and the 5th, 50th, and 95th percentiles of the total knowledge score and both subscores, for all specialists, in percentages. As can be seen from comparison of the confidence intervals provided in Table 2, the means of the total knowledge score and both subscores differed statistically significantly between the CGs and the other specialties $(P<.0001)$, as well as between the respective nongeneticists $(P<.0001)$, except that there was no significant difference in the "fact score" between the GYNs and the PEDs $(P=1.0)$. The mean knowledge scores of the GPs were lower than those of the GYNs, which were lower than those of the PEDs. Furthermore, the variation in individual scores was highest among the GPs; the 5th percentile of GPs, GYNs, and PEDs, respectively, was at approximately 40\%, 52\%, and 62\% correct answers.

Table 2

Total knowledge and subscores for different groups of respondents

\begin{tabular}{cccc}
\hline GPs & GYNs & PEDs \\
\hline & correct
\end{tabular}

Total Knowledge Score

\begin{tabular}{lcccc} 
Mean & 64 & 75 & 81 & \multicolumn{1}{c}{95} \\
$95 \% \mathrm{CI}^{b}$ & $61-66$ & $73-76$ & $79-82$ & $94-96$ \\
SD & 13.9 & 11.7 & 10.1 & 4.2 \\
Percentiles & & & & \\
5 & 39.6 & 51.5 & 61.5 & 88.5 \\
50 & 64.6 & 76.9 & 80.8 & 96.2 \\
95 & 84.6 & 90.4 & 92.3 & 100 \\
"Fact Score" & & & & \\
Mean & 55 & 70 & 71 & 90 \\
$95 \% \mathrm{CI}^{b}$ & $52-59$ & $68-73$ & $69-74$ & $88-94$ \\
SD & 19.6 & 15.9 & 17.3 & 10.0
\end{tabular}

Percentiles

5

5

50

20.0

44.4

42.2

77.8

95

55.6

72.2

75.6

88.9

"Concept Score"

Mean

95\% $\mathrm{CI}^{b}$

83.3

88.9

88.9

100

$\mathrm{SD}$

\begin{tabular}{ll}
$68 \quad 7$ \\
\hline
\end{tabular}

77

86

97

65-71

75-79

84-88

$96-98$

16.4

13.6

10.5

3.8

Percentiles

\begin{tabular}{lcccc}
5 & 35.3 & 52.9 & 64.7 & 88.2 \\
50 & 70.6 & 82.4 & 88.2 & 100 \\
95 & 94.1 & 94.1 & 100 & 100 \\
\hline
\end{tabular}

${ }^{a}$ Scores used for validation.

${ }^{b}$ of the mean.

GP, general practitioner; GYN, gynecologist; PED, pediatrician; CG, clinical geneticist; CI, confidence interval; SD, standard deviation. 


\section{Baars et al.}

To describe the answers on the questionnaire, a selected number of aspects will be summarized in detail. Thirty-four percent of the GPs, $8 \%$ of the GYNs, and $4 \%$ of the PEDs answered that there was no available DNA or biochemical test for CF. The question about male infertility being a characteristic of CF was incorrectly answered by $49 \%, 23 \%$, and $8 \%$ of the GPs, GYNs, and PEDs, respectively. Half of the GPs, $37 \%$ of GYNs, and $21 \%$ of PEDs could not correctly calculate the chance of having a child with CF after being told the carrier frequency. Seventy-four percent of the GPs, $50 \%$ of the GYNs, and $46 \%$ of the PEDs were unaware of the availability of DNA or biochemical tests for sickle-cell anemia. Forty-seven percent of the GPs, $41 \%$ of the GYNs, and $15 \%$ of the PEDs were unable to recognize an autosomal dominant inheritance pattern in a pedigree.

\section{Factors associated with the total knowledge scores}

Table 3 shows the results of multivariate regression analysis of the factors associated with the knowledge score for each of the specialties. For GPs, year of graduation and an elective course in genetics were positively associated with their knowledge score. A (very) densely urbanized practice location was negatively associated with their knowledge score. Variables significantly associated with the knowledge scores of the GYNs were year of graduation, involvement in fellows education, provision of genetic counseling in their own practice, and attendance at meetings outside their own region to keep their genetic knowledge up to date. Variables positively associated with the knowledge scores of the PEDs were an elective course in genetics, involvement in fellows education, and provision of genetic counseling in their own practice.

\section{DISCUSSION}

The objective of this study was to investigate knowledge of genetics and awareness of genetic tests among GPs, GYNs, and
PEDs in The Netherlands. The level of genetic knowledge among these nongeneticists was lower than that in the CG validation group, as expected. The variation in individual scores was highest among the GPs; the 5th percentile of GPs, GYNs, and PEDs was at approximately $40 \%, 52 \%$, and $62 \%$ correct answers, respectively, and the 95th percentile of GPs, GYNs, and PEDs was at approximately $85 \%, 90 \%$, and $92 \%$ correct answers, respectively. The questions, designed by a panel including nongeneticists, are considered to be important for counseling in primary care. ${ }^{5}$ Nevertheless, the knowledge scores of many nongeneticists reflected clear deficiencies.

\section{Factors associated with knowledge}

First a comparison of our results with the study carried out by Hofman et al. ${ }^{5} 10$ years ago is described. Next our results will be compared with other studies. As also shown in the U.S. study, knowledge scores in our study were influenced by specialty (GP, GYN, or PED). In addition, physicians who more recently graduated had a higher knowledge score, which is in line with findings in the United States. More involvement in obstetrics was, in contrast with the findings in the United States, not related to a higher knowledge score among the GPs. ${ }^{5}$ This may be related to the small number of GPs who delivered babies: $14 \%$ compared with $29 \%$ in the U.S. study. In addition, attending a required course in genetics did not influence the knowledge score, as it did in the United States. ${ }^{5,13}$ More interest in genetics, approximated by taking an elective course in genetics, was related to a higher knowledge score among the GPs and PEDs, which was in contrast with the results of the study in the United States. ${ }^{5}$ Full-time faculty members did not score, in contrast with the findings in the United States, a higher knowledge score. ${ }^{5}$ Moreover, working in a university hospital also had no influence on the knowledge of the PEDs or GYNs in our study. The negative correlation between the level of urbanization of the practice and the knowledge

Table 3

Multiple regression analysis of variables associated with total knowledge scores

\begin{tabular}{|c|c|c|c|c|c|c|}
\hline \multirow[t]{2}{*}{ Predictors } & \multicolumn{2}{|c|}{ GPs } & \multicolumn{2}{|c|}{ GYNs } & \multicolumn{2}{|c|}{ PEDs } \\
\hline & $\beta$ & p-value & $\beta$ & p-value & $\beta$ & p-value \\
\hline Year of graduation & 0.264 & 0.00 & 0.181 & 0.01 & 0.120 & 0.11 \\
\hline Elective course in genetics & 0.208 & 0.02 & 0.106 & 0.14 & 0.175 & 0.02 \\
\hline (Very) dense urbanisation & -0.224 & 0.01 & NA & & NA & \\
\hline Involvement in fellow education & NS & & 0.167 & 0.02 & 0.156 & 0.04 \\
\hline Providing genetic counselling & 0.151 & 0.09 & 0.264 & 0.00 & 0.234 & 0.00 \\
\hline $\begin{array}{l}\text { Information source: meetings } \\
\text { outside the region }\end{array}$ & NS & & 0.214 & 0.00 & NS & \\
\hline Course genetics in curriculum & -0.149 & 0.53 & -0.057 & 0.46 & NS & \\
\hline Attended required course in genetics & 0.311 & 0.19 & NS & & NS & \\
\hline
\end{tabular}

$$
\mathrm{R}^{2}=0.18
$$$$
\mathrm{R}^{2}=0.19
$$$$
\mathrm{R}^{2}=0.13
$$

GP, general practitioner; GYN, gynecologist; PED, pediatrician; NA, not applicable; NS, not significant in univariate linear regression analysis $(P>.10)$. 
score is possibly linked to higher workload, because of a shortage of GPs in the cities. Another speculation could be that urban GPs can refer to specialist care much more readily and therefore need less knowledge than their rural colleagues. No association between level of urbanization and knowledge was found in the United States. ${ }^{5}$ In the U.S. study, country of medical school influenced the knowledge score, but in The Netherlands, where $97 \%$ of the respondents attended a Dutch medical school, no association was found, probably because of a lack of variance. ${ }^{5}$ In our study, innovativeness (physicians who were using a new predictive test earlier than it became standard practice) was, in contrast with findings in the United States, not associated with a higher knowledge score. ${ }^{5}$

Low exposure to genetic problems, as in general practice, was an important predictor of lack of genetic knowledge, comparable to the findings of other studies. ${ }^{5,6,8,9,14-16}$ No association was found between gender and knowledge, which was in line with findings in a Swiss study among GPs, internists, GYNs, and oncologists. ${ }^{8}$ Physicians who more recently graduated had a higher knowledge score, which is in line with findings in another study in the United State ${ }^{13}$ but in contrast with the results of a Swiss study, in which age was not found to be a predictor. $^{8}$

Only a limited amount of the variance $(13 \%-19 \%)$ in the knowledge scores was explained by the variables studied. Other factors, not measured in this questionnaire, are probably also important, such as the amount of time allocated to genetic education in the medical curriculum.

\section{Deficiency of genetic knowledge and its impact}

The deficiencies in knowledge of genetics among a large percentage of physicians that were found in this study are in line with the findings of other studies. ${ }^{5-9}$ In several countries, including The Netherlands, referrals to specialists are mainly controlled by the GPs, in their role as gatekeepers to specialist care. ${ }^{4}$ Sikkens et al. ${ }^{17}$ reported that the rate of referral for genetic counseling of couples with a child with a congenital disorder did not improve over the years. Furthermore, approximately $10 \%$ to $20 \%$ of the women with a known genetic risk factor did not attend a genetic center before they were pregnant, and the main reason for referral by the GP during pregnancy instead of before was the GP's unawareness of the potential risk factor. ${ }^{18}$ Knowledge of genetic tests is shown to be a positive predictor for referral to genetic centers. ${ }^{6}$ In view of the increasing interest and diagnostic options in genetics, and increasing demands from the public, a higher level of knowledge among physicians is required. Several studies have shown that primary care providers are willing to incorporate more genetics in their practice in the future, but that they express a need for more education in genetics. ${ }^{7,19-21}$ The percentage of correct answers on the concept score was higher than that of those on the fact score, and this applied for each specialty. The higher "concept" knowledge is advantageous because the concept questions give more insight in the responders' understanding of the complexity of genetics. Specific attention should be paid to the fact that deficiencies in knowledge found in our sample of physicians is similar to the deficiencies found by Hofman et al. ${ }^{5} 10$ years ago. This is in line with the findings of an earlier study, which already demonstrated that without intervention there was no change in the attitude of GPs toward genetics. ${ }^{22}$ One could argue that despite the willingness of physicians and the call for more education, educational programs probably did not focus on the right topics or attendees. More attention should be paid to continued educational programs, for older physicians and GPs in particular. In a study carried out by Qureshi et al. ${ }^{23}$ the participating GPs stated that their preference would be an educational program including joint hospital/general practice guidelines and meetings. The family physicians participating in the study carried out by Fetters et al. ${ }^{19}$ wanted to know more about educational topics, who needs genetic counseling, and what can be expected from genetic counselors. It was argued that taking, recording, and interpreting family history should be of major importance for primary care providers. ${ }^{24,25}$ Starfield et al. ${ }^{26}$ presented a few simple aspects that are important in this respect, such as prevalence of the symptoms, family history, ethnic background, and persistence of symptoms, and indicated that these would improve the recognition of genetic problems in primary care. Courses on specific genetic topics organized by professional societies are reported to be effective in increasing the knowledge of the specialists. ${ }^{16}$ A research project among medical students nearing graduation recently showed that the students lacked the appropriate knowledge of genetics that is relevant for daily practice, and that changes in the basic medical curriculum should be made as well. ${ }^{27}$ Furthermore, as demonstrated by Challen et al. ${ }^{28}$ the integration and visibility of genetics in the medical curricula in several countries in Europe was very limited.

\section{Limitations of the study}

It is impossible to rule out the possibility that some respondents removed the stickers covering the additional information before answering the preceding questions. This implies that the knowledge scores could be inflated. However, the knowledge was measured in large samples of physicians and with sufficient response rates, and therefore the calculated differences in the knowledge scores should reflect clear differences in the knowledge between the various specialties. ${ }^{29}$ In some countries the GPs do not have the role of "gatekeeper," because of differences in the health care systems, and therefore the results of this study should be extrapolated to other countries with caution. In contrast with our expectations, no significant influence of SCID membership was found on the knowledge scores of the PEDs. Only a small number $(n=36)$ of questionnaires of SCID members could be analyzed, and for that reason our study simply could have not enough power to demonstrate a difference in knowledge scores.

\section{CONCLUSIONS}

In conclusion, the level of knowledge of many of the participating physicians was found to be too low to guarantee an 


\section{Baars et al.}

adequate response to questions patients ask about genetics and genetic tests and to new developments in the field of genetics. This lack of genetic knowledge is, as far as we know now, a global problem. A solution to increase genetic knowledge, specifically pedigree analysis, the interpretation of probabilistic results, and the availability of an expanding number of DNA tests, among physicians could be the formation of a coalition between stakeholders. ${ }^{30}$

\section{ACKNOWLEDGMENTS}

We thank all the GPs, GYNs, PEDs, and CGs who participated in the survey. We also thank N.A. Holtzman for giving us permission to use the questionnaire and for his critical review of the article. We thank M.C. Cornel and G.A. van Essen for their helpful comments on earlier drafts of this article, and to M.W. Langendam and H.J. Adèr for their advice on statistical analysis. This research was supported by the Foundation for Clinical Genetics Amsterdam, The Netherlands.

\section{References}

1. Collins FS, McKusick VA. Implications of the Human Genome Project for medical science. JAMA 2001;285:540-544.

2. Bell J. The new genetics in clinical practice. BMJ 1998;316:618-620.

3. Mathew C. Science, medicine, and the future: postgenomic technologies: hunting the genes for common disorders. BMJ 2001;322:1031-1034.

4. Boerma WG, Van der Zee J, Fleming DM. Service profiles of general practitioners in Europe. European GP Task Profile Study. Br J Gen Pract 1997;47:481-486.

5. Hofman KJ, Tambor ES, Chase GA, Geller G, Faden RR, Holtzman NA. Physicians' knowledge of genetics and genetic tests. Acad Med 1993;68:625-632.

6. Hunter A, Wright P, Cappelli M, Kasaboski A, Surh L. Physician knowledge and attitudes towards molecular genetic (DNA) testing of their patients. Clin Genet 1998;53:447-455.

7. Watson EK, Shickle D, Qureshi N, Emery J, Austoker J. The 'new genetics' and primary care: GPs' views on their role and their educational needs. Fam Pract. 1999;16:420-425.

8. Escher M, Sappino AP. Primary care physicians' knowledge and attitudes towards genetic testing for breast-ovarian cancer predisposition. Ann Oncol 2000;11:11311135.

9. Schroy PC, Barrison AF, Ling BS, Wilson S, Geller AC. Family history and colorectal cancer screening: a survey of physician knowledge and practice patterns. Am J Gastroenterol 2002;97:1031-1036.

10. Suther SG, Goodson P. Barriers to the provision of genetic services by primary care physicians: a systematic review of the literature. Genet Med 2003;5:70-76.

11. Baars MJH, Henneman L, Ten Kate LP. Preconceptional cystic fibrosis carrier screening: opinions of general practitioners, gynecologists, and pediatricians in the Netherlands. Genet Test 2004;8:431-436.
12. Geller G, Tambor ES, Chase GA, Holtzman NA. Measuring physicians' tolerance for ambiguity and its relationship to their reported practices regarding genetic testing. Med Care 1993;31:989-1001.

13. Wilkins-Haug L, Hill LD, Power ML, Holzman GB, Schulkin J. Gynecologists' training, knowledge, and experiences in genetics: a survey. Obstet Gynecol 2000;95:421424 .

14. Acton RT, Burst NM, Casebeer L, Ferguson SM, et al. Knowledge, attitudes, and behaviors of Alabama's primary care physicians regarding cancer genetics. Acad Med 2000;75:850-852.

15. Firth HV, Lindenbaum RH. UK clinicians' knowledge of and attitudes to the prenatal diagnosis of single gene disorders. J Med Genet 1992;29:20-23.

16. Doksum T, Bernhardt BA, Holtzman NA. Does knowledge about the genetics of breast cancer differ between nongeneticist physicians who do or do not discuss or order BRCA testing? Genet Med 2003;5:99-105.

17. Sikkens EH, de Walle HE, Reefhuis J, vanTintelen JP, van Essen AJ. Referral for genetic counseling after the birth of a child with a congenital anomaly in the Northern Netherlands. Am J Med Genet 2002;112:133-137.

18. Aalfs CM, Smets EM, de Haes HC, Leschot NJ. Referral for genetic counselling during pregnancy: limited alertness and awareness about genetic risk factors among GPs. Fam Pract 2003;20:135-141.

19. Fetters MD, Doukas DJ, Phan KL. Family physicians' perspectives on genetics and the human genome project. Clin Genet 1999;56:28-34.

20. Emery J, Watson E, Rose P, Andermann A. A systematic review of the literature exploring the role of primary care in genetic services. Fam Pract 1999;16:426-445.

21. Suchard MA, Yudkin P, Sinsheimer JS, Fowler GH. General practitioners' views on genetic screening for common diseases. Br J Gen Pract 1999;49:45-46.

22. Baars MJH, de Smit DJ, Langendam MW, Ader HJ, ten Kate LP. Comparison of activities and attitudes of general practitioners concerning genetic counseling over a 10-year time-span. Patient Educ Couns 2003;50:145-149.

23. Qureshi N, Hapgood R, Armstrong S. Continuous medical education approaches for clinical genetics: a postal survey of general practitioners. J Med Genet 2002;39: e69.

24. Emery J, Hayflick S. The challenge of integrating genetic medicine into primary care. BMJ 2001;322:1027-1030.

25. Burke W, Emery J. Genetics education for primary-care providers. Nat Rev Genet 2002;3:561-566.

26. Starfield B, Holtzman NA, Roland MO, Sibbald B, Harris R, Harris H. Primary care and genetic services. Health care in evolution. Eur J Public Health 2002;12:51-56

27. Baars MJH, Scherpbier AJJA, Shuwirth LW, Henneman L, et al. Deficient knowledge of genetics relevant for daily practice among medical students nearing graduation. Genet Med 2005;7:295-301.

28. Challen K, Harris HJ, Julian-Reynier C, Ten Kate LP, et al. Genetic education and non-genetic health professionals: educational providers and curricula in Europe. Genet Med 2005;7:302-310.

29. Tambor ES, Chase GA, Faden RR, Geller G, Hofman KJ, Holtzman NA. Improving response rates through incentive and follow-up: the effect on a survey of physicians' knowledge of genetics. Am J Public Health 1993;83:1599-1603.

30. Core Competency Working Group of the National Coalition for Health Professional Education in Genetics. Recommendations of core competencies in genetics essential for all health professionals. Genet Med 2001;3:155-159. 